\title{
Band of Critical States in Anderson Localization in a Strong Magnetic Field with Random Spin-Orbit Scattering
}

\author{
C. Wang, ${ }^{1,2}$ Ying Su, ${ }^{1,2}$ Y. Avishai, ${ }^{3,{ }^{*}}$ Yigal Meir, ${ }^{3}$ and X. R. Wang ${ }^{1,2, \dagger}$ \\ ${ }^{1}$ Physics Department, The Hong Kong University of Science and Technology, Clear Water Bay, Kowloon, Hong Kong \\ ${ }^{2}$ HKUST Shenzhen Research Institute, Shenzhen 518057, China \\ ${ }^{3}$ Department of Physics, Ben-Gurion University of the Negev, Beer-Sheva, Israel
}

(Received 18 November 2014; published 5 March 2015)

\begin{abstract}
The Anderson localization problem for a noninteracting two-dimensional electron gas subject to a strong magnetic field, disordered potential, and spin-orbit coupling is studied numerically on a square lattice. The nature of the corresponding localization-delocalization transition and the properties of the pertinent extended states depend on whether the spin-orbit coupling is uniform or fully random. For uniform spin-orbit coupling (such as Rashba coupling due to a uniform electric field), there is a band of metallic extended states in the center of a Landau band as in a "standard" Anderson metal-insulator transition. However, for fully random spin-orbit coupling, the familiar pattern of Landau bands disappears. Instead, there is a central band of critical states with definite fractal structure separated at two critical energies from two side bands of localized states. Moreover, finite size scaling analysis suggests that for this novel transition, on the localized side of a critical energy $E_{c}$, the localization length diverges as $\xi(E) \propto \exp \left(\alpha / \sqrt{\left|E-E_{c}\right|}\right)$, a behavior which, together with the emergence of a band of critical states, is reminiscent of a Berezinskii-Kosterlitz-Thouless transition.
\end{abstract}

DOI: 10.1103/PhysRevLett.114.096803

PACS numbers: 73.20.Fz, 71.30.+h, 73.43.Nq

Traditionally, noninteracting disordered electronic systems subject to a random potential are classified according to the symmetries of their Hamiltonian under time reversal (TR) and spin rotation (SR) transformations. Considering the Hamiltonian as a random matrix [1-3], its symmetries determine to which random matrix Gaussian ensemble (or universality class) it belongs, orthogonal (TR and SR satisfied), symplectic ( SR violated), or unitary (TR violated).

This classification is intimately related to one of the most fundamental concepts in the physics of disordered electronic systems: the Anderson localization transition (ALT) $[4,5]$, which is a quantum phase transition between localized and extended states in a disordered system. The critical dimension for existence or nonexistence of ALT is $d=2$. For $d<2$ there is no ALT, while for $d>2$ there is always ALT. Hence, for a two-dimensional electron gas (2DEG), ALT (if it exists) is of special interest. The scaling theory [6] together with calculations based on the nonlinear sigma model $[7,8]$ established that for $d=2$ ALT does not exist for the orthogonal and unitary classes (zero or finite magnetic field, respectively), and does exist for the symplectic class [finite spin-orbit coupling (SOC) and zero magnetic field]. Later, topology was also recognized as a property determining the pertinent universality class [10]. In the presence of a topological term in the action of the corresponding nonlinear sigma model [11], it was established that if SR invariance is respected, the system is in the IQHE universality class characterized by a Hall transition between localized and critical states occurring at discrete energies. What happens in the presence of the topological term when SR invariance is broken is still unclear. To the best of our knowledge, there is so far no rigorous extension of the nonlinear sigma model for an IQHE system in the presence of strong SOC.

In this work, we investigate the nature of transition between localized and extended states for a disordered 2DEG subject to a strong magnetic field in which SR invariance is broken due to SOC. In Ref. [12], the effect of random SOC between the two lowest Landau levels was shown to lead to split percolation transitions. Here we show that the nature of the SOC (uniform or fully random) dramatically affects the pertinent transition. Our main results are as follows: (1) For uniform SOC, the pattern of separated Landau bands persists. Focusing attention on the lowest Landau band entered at $E_{0}$, there are two critical energies, $E_{c_{1}}$ and $E_{c_{2}}$, in the sense that the states $\psi_{E}(\mathbf{r})$ for $E_{c 1}<E<E_{c 2}$ are metallic, while for $E \notin\left[E_{c_{1}}, E_{c_{2}}\right]$, they are localized. This is a "usual" ALT between a band of localized states and a band of metallic states. (2) However, for fully random spin-orbit coupling, the structure of broadened Landau bands that is the hallmark of the IQHE is completely washed out. In turn, there is a broad band of critical states with definite fractal structure (of dimension $1.82 \pm 0.02$ ). Finite size scaling analysis suggests that for this novel transition, the localization length diverges as $\xi(E) \propto \exp \left(\alpha / \sqrt{\left|E-E_{c}\right|}\right)$, a behavior reminiscent of a Berezinskii-Kosterlitz-Thouless (BKT) transition [13].

To substantiate our claims we consider a tight-binding Hamiltonian for 2DEG on a square lattice of length $L$ and width $M$ (the lattice constant is set to unity), 
$H=\sum_{i, \sigma} \epsilon_{i} c_{i, \sigma}^{\dagger} c_{i, \sigma}+\sum_{\langle i j\rangle, \sigma, \sigma^{\prime}} \exp \left(i \phi_{i j}\right) V_{i j}\left(\sigma, \sigma^{\prime}\right) c_{i, \sigma^{\prime}}^{\dagger} c_{j, \sigma}$.

Here, $i=\left(n_{i}, m_{i}\right)$ is a lattice site specified by integer coordinates $n_{i}$ and $m_{i}$ with $1 \leq n_{i} \leq L$ and $1 \leq m_{i} \leq M$. $c_{i, \sigma}^{\dagger}\left(c_{i, \sigma}\right)$ is electron creation (annihilation) operator of spin $\sigma= \pm$ on site $i$. The on-site energy $\epsilon_{i}$ are random and uniformly distributed in the range of $[-W / 2, W / 2]$, so that $W$ measures the degree of randomness. The symbol $\langle i j\rangle$ indicates that $i$ and $j$ are nearest neighbor sites. The magnetic field is introduced through the Peierls' substitution [14] by endowing the hopping coefficient with a phase, $\phi_{i j}=(e / \hbar) \int_{i}^{j} \vec{A} \cdot d \vec{l}$, where $\vec{A}$ is the vector potential. A constant magnetic field $B$ is expressed as magnetic flux in units of $\Phi_{0}=c h / e$ through a unit square.

The SOC is encoded by $2 \times 2$ matrices $V_{i j}$ acting in spin space. We will explore both the case of constant SOC matrices along the axes and the case of fully random SOC matrices. In the case of constant SOC matrices, they are parametrized as $V_{i j}=V_{x}\left(V_{y}\right)$ for $\langle i j\rangle$ along the $x$ direction ( $y$ direction). In order to get nontrivial results due to SOC, one requires $\left[V_{x}, V_{y}\right] \neq 0$.

The Rashba form of uniform SOC reads

$$
V_{x}=\left[\begin{array}{cc}
1 & a \\
-a & 1
\end{array}\right] \text { and } V_{y}=\left[\begin{array}{cc}
1 & -i a \\
-i a & 1
\end{array}\right]
$$

where $a$ is a real constant encoding the strength of the SOC for the Rashba model. Random SOC is encoded by matrices $V_{i j} \in \mathrm{SU}(2)$, thereby defining the $\mathrm{SU}(2)$ model [15],

$$
V_{i j}=\left[\begin{array}{ll}
e^{-i \alpha_{i j}} \cos \left(\beta_{i j} / 2\right) & e^{-i \gamma_{i j}} \sin \left(\beta_{i j} / 2\right) \\
-e^{i \gamma_{i j}} \sin \left(\beta_{i j} / 2\right) & e^{i \alpha_{i j}} \cos \left(\beta_{i j} / 2\right)
\end{array}\right],
$$

where $\alpha_{i j}$, and $\gamma_{i j}$ are uniformly distributed in $[0,2 \pi]$ and $\sin \left(\beta_{i j} / 2\right)$ is uniformly distributed in [0,1]. Such a random SOC model can mimic 2DEGs of semiconductor heterostructures with charged impurities (donors or acceptors) [16], and has been successfully applied to describe weak localization effects in, e.g., GaAs/AlGaAs wells [17]. Interestingly, this model can also be mapped onto a BCS model, thereby describing dirty superconductors [18].

Localization-delocalization transition in an electronic system is characterized not only by divergence of the localization length but also by the nature of the wave functions $\psi_{E_{c}}(\mathbf{r})$ at the critical energies. Analysis of these two independent criteria usually requires two different numerical procedures.

Identification of ALT.-We consider a scattering problem for an electron at Fermi energy $E$ living on a square lattice of length $L \rightarrow \infty$ (along $x$ ) and finite width $M$ (along $y$ ). Periodic boundary conditions are imposed along $y$ to avoid edge states contribution.
Since the system is quasi one-dimensional, it has a finite localization length $\lambda_{M}(E)$ depending on the scattering energy $E$ and the system's width $M$. Using the transfer matrix technique we calculate $\lambda_{M}(E)$ by a standard iteration algorithm $[4,19]$. In our calculations $L>10^{6} \gg \lambda_{M}(E)$ and self-averaging is virtually achieved, requiring averaging over a small number of realizations to achieve good statistics. The width $M$ takes values between 32 and 96 . The identification of a mobility edge $E_{c}$ is guided by the following observations: (1) For metallic (insulating) states, $\bar{\lambda}_{M}(E) \equiv$ $\lambda_{M}(E) / M$ is an increasing (decreasing) function of $M$. (2) For critical states, $\bar{\lambda}_{M}(E)$ is independent of $M$. (3) For energy $E$ close to $E_{c}$ but on the insulating side, $\bar{\lambda}_{M}(E)$ obeys a single parameter finite size scaling. Explicitly, let us denote by $\xi(E) \equiv \lambda_{\infty}(E)$ the localization length for a system of width $M \rightarrow \infty$. At the critical energy we expect $\xi\left(E_{c}\right)=\infty$, and finite size scaling implies $\bar{\lambda}_{M}(E)=f[M / \xi(E)]$, where $f(x)$ is a universal (disorder independent) scaling function.

Results for constant SOC, Eq. (2).--In this case, the pattern of separate LBs remains intact, and our attention is focused on the lowest LB. The upper panel of Fig. 1(a) displays $\bar{\lambda}_{M}(E)$ vs $E$ for $B=1 / 5$ and $W=1$ in the absence of the SOC $(a=0)$. It is evident that all curves for different widths $M$ coalesce at $E_{c}$, where $\bar{\lambda}_{M}\left(E_{c}\right)$ peak. On both sides of $E_{c}$, all states are localized because $\bar{\lambda}_{M}(E)$ decreases with $M$. However, at the critical point $E_{c}, \bar{\lambda}_{M}(E)$ is independent of $M$, and the corresponding states are critical [20]. The
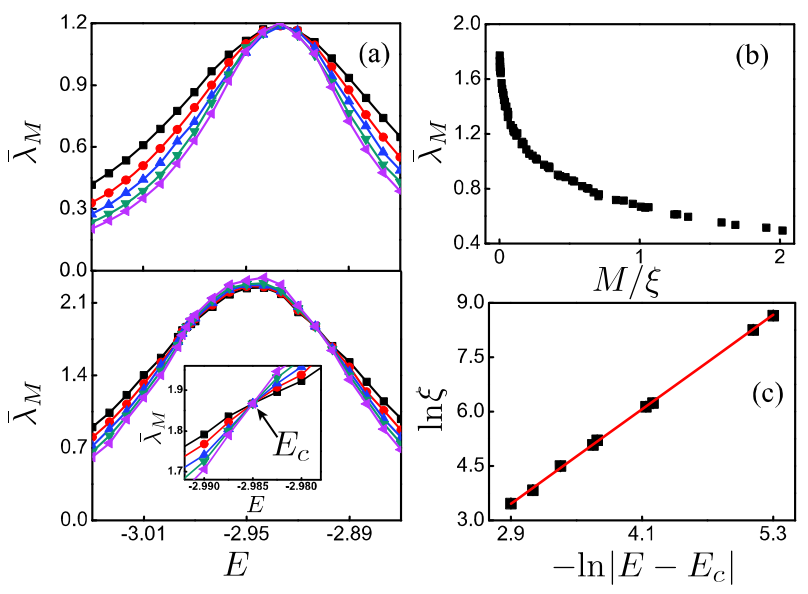

FIG. 1 (color online). $\bar{\lambda}_{M}(E)$ (averaged over 40 disorder realizations), vs $E$ for $B=1 / 5$ and $W=1$. (a) Without SOC (upper panel) and with constant Rashba SOC, Eq. (2) with $a=$ 0.1 (bottom panel). The system widths (top to bottom) are $M=$ 32 (square), 48 (circle), 64 (up triangle), 80 (down triangle), and 96 (left triangle). The inset at the bottom panel is an enlargement of the crossing region around $E_{c}=-2.985$. (b) When the points $\bar{\lambda}_{M}(E)$ shown in (a) are expressed in terms of $x=M / \xi(E)$, they fall on a smooth curve thereby display the scaling function. (c) $\ln \xi(E)$ vs $\ln \left(\left|E-E_{c}\right|\right), E_{c}=-2.985$, for the constant Rashba SOC. The solid line is a linear fit with slope $\nu=2.2$. 
bottom panel displays $\bar{\lambda}_{M}(E)$ for the same values of $B$ and $W$ as in the upper panel when the constant (Rashba) SOC strength is $a=0.1$. Here, in contradistinction from the case of zero SOC, curves of different $M$ cross at two energies $\left(E_{c, 1} \simeq-2.91, E_{c, 2} \simeq-2.985\right)$. States $\psi_{E}(\mathbf{r})$ for $E \in$ $\left[E_{c, 1}, E_{c, 2}\right]$ are metallic because $\bar{\lambda}_{M}(E)$ increases with $M$, indicating that these are extended states.

Now we employ a finite size one-parameter scaling hypothesis to substantiate the criticality of the transition, by showing that for $E$ close to $E_{c, i}, \bar{\lambda}_{M}(E)=f[M / \xi(E)]$. The results are summarized in Figs. 1(b) and 1(c). As shown in Fig. 1(b), in the vicinity of the crossing points, all data points $\bar{\lambda}_{M}(E)$ for the Rashba SOC case collapse on to a single smooth scaling curve $f(x)$. Like in the standard ALT or Hall transitions, $\xi(E)$ diverges at $E_{c}$ as a power, $\left|E-E_{c}\right|^{-\nu}$. This is substantiated in Fig. 1(c) that displays $\ln \xi(E)$ vs $\ln \left|E-E_{c, 2}\right|$ for the Rashba SOC case, Eq. (2). The fit to a straight line is rather satisfactory, yielding a slope $\nu_{1}=2.2 \pm 0.1$ that is somewhat smaller than both critical exponents of the 2D disordered systems $\nu \simeq 2.75$ for the 2D symplectic symmetry class [15] and $\nu \simeq 2.34$ for the IQHE [5].

Results for the random SU(2) model, Eq. (3).- -In the absence of a magnetic field, the SU(2) model supports a standard ALT. This is substantiated in the upper panel of Fig. 2(a) which plots $\ln \left[\bar{\lambda}_{M}(E)\right]$ vs $E$ for $W=1$ and various $M$. All curves cross at $E_{c}=-3.259$, showing all states of $E \in[-3.259,3.259]$ are extended because $\ln \left[\bar{\lambda}_{M}(E)\right]$ increases with $M$ as shown clearly in the inset of the enlarged crossing region. Finite size scaling yields the value $\nu=$ $2.73 \pm 0.02$ commensurate with earlier calculations [15].

We come now to the main result of the present work. Switching on a strong magnetic field (here we take $B=1 / 5$ ) one would expect a pattern of LB modified due to the presence of SOC. However, what we find is that the curves $\bar{\lambda}_{M}(E)$ do not display separate LB peaks, but, rather, a single band. More remarkably, in contradistinction with the symplectic case (SOC $\neq 0$ and $B=0$ ), the curves $\bar{\lambda}_{M}(E)$ that display a localized region for energies $\{E\}$ near the band edge [that is, $\bar{\lambda}_{M}(E)$ decreases with $M$ ], do not cross but merge as the energy approaches the band center. This is evident by looking at the bottom panel of Fig. 2(a) that displays $\ln \left[\bar{\lambda}_{M}(E)\right]$, averaged over 40 ensembles, as a function of $E$ for $B=1 / 5, W=1$ and various system widths $M$. The inset is an enlargement of the merging region. For $E<E_{c}=-3.001$ the system behaves as an insulator where $\bar{\lambda}_{M}(E)$ decreases with $M$. But for $E \geq E_{c}$ all curves merge, forming a band of critical states for which $\bar{\lambda}_{M}(E)$ is independent of $M$, and, as we shall see below, the corresponding wave-functions cover only a fractal part of 2D space. This band of critical states prevails for all energies $|E|<\left|E_{c}\right|=3.001$, namely, the pattern of separate Landau bands is completely washed out.
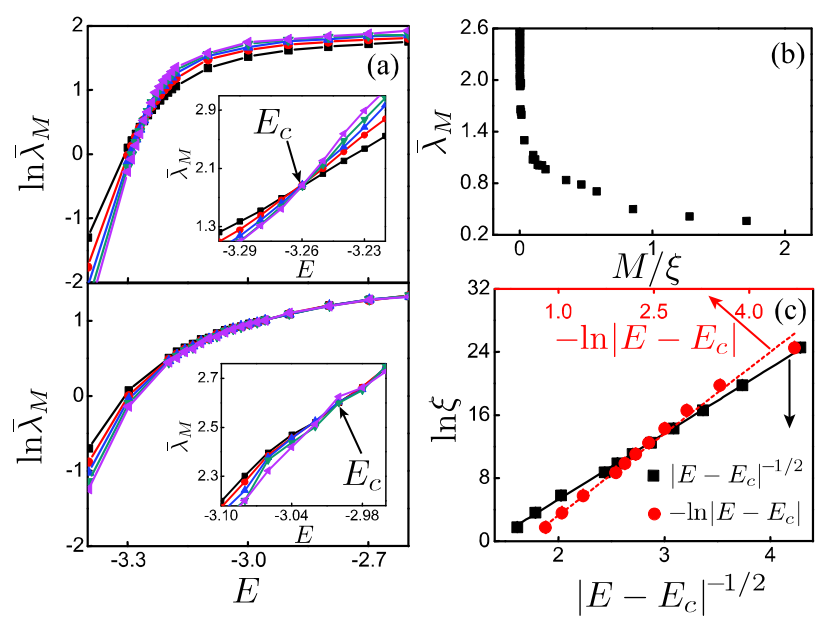

FIG. 2 (color online). (a) $\ln \left(\bar{\lambda}_{M}\right)$ vs $E$ of the $S U(2)$ model with $W=1$ and $M=32$ (square), 48 (circle), 64 (up triangle), 80 (down triangle), and 96 (left triangle). The top panel is for $B=0$, and the inset is an enlargement of the crossing region in linear scale. The bottom panel is for $B=1 / 5$, and the inset is an enlargement of the merging region. (b) The scaling function obtained from the bottom panel in (a) by collapsing data of $\bar{\lambda}_{M}$ near the merging point into a single curve. (c) $\ln \xi$ vs $\left|E-E_{c}\right|^{-1 / 2}$ for $E_{c}=-3.001$ (squares). The solid line is a linear fit with slope $\alpha=8.3 \pm 0.3$. For a comparison, $\ln \xi$ vs $-\ln \left|E-E_{c}\right|$ with $E_{c}=$ -3.051 (circles) is also plotted. Larger deviation in the linear fit (dashed line with goodness of fit of $5.2 \times 10^{-7}$ ) indicates that an interpretation in terms of BKT-type transition (with goodness of fit of $8 \times 10^{-3}$ ) explains the data better.

To explore the nature of this localization-delocalization transition we inspect the behavior of $\xi(E)$ on the insulating side $E<E_{c}$. Figure 2(b) depicts the collapse of all curves $\bar{\lambda}_{M}(E)$ for different widths $M$, supporting the quantum phase transition interpretation. However, if one analyzes the divergence of $\xi$ in terms of a power law, as shown by the red dotted line in the log-log plot in Fig. 2(c), the goodnessof-fit [21] to the numerical data (red circles) is about $5.2 \times 10^{-7}$, which is 4 orders of magnitude smaller than an acceptable value. Moreover, the resulting exponent turns out to be $\nu \simeq 6.4$, much higher than any known critical exponent in disordered 2D systems. We argue that the transition between bands of localized and critical states exposed here is reminiscent of a BKT phase transition [13], where $\xi(E) \propto \exp \left(\alpha / \sqrt{\left|E_{c}-E\right|}\right)$. Indeed, the curve displaying $\ln \xi$ vs $1 / \sqrt{E-E_{c}}$ in Fig. 2(c) is well fitted by a straight line, the corresponding goodness of fit to the numerical data (black squares) is about $8 \times 10^{-3}$, far better than the power-law fit, thereby supporting our claim pertaining to a BKT-type transition [19].

Fractal structure of critical states.-To confirm the criticality of all states at energies $|E|<E_{c}$, we compute, by exact diagonalization, the normalized electron wave functions $\psi_{E}(i)$ on a square lattice of finite extent [for convenience we take a rectangle of area $M(M+1)]$. Here 

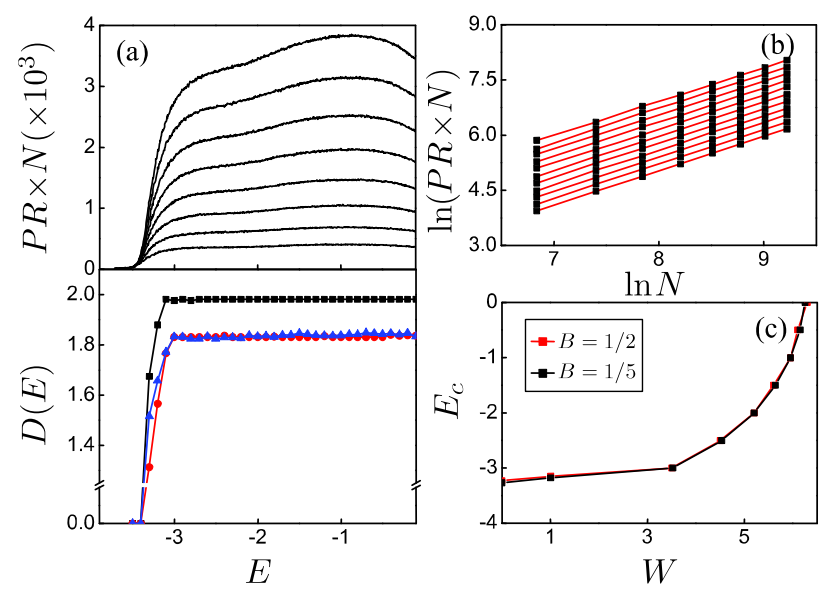

FIG. 3 (color online). (a) Upper panel: $P R(E) \times N$, averaged over 100 samples, as a function of $E$ of the SU(2) model for $B=1 / 5$ and $W=1$. The lattice size (from down up) is $M=30$, $40,50,60,70,80,90$, and 100. Lower panel: $D(E)$ vs $E$ for $W=1$ and $B=0$ (black square); $B=1 / 5$ (red circle); $B=1 / 2$ (blue triangle). (b) The quantity $\ln [P R(E) \times N]$ is plotted vs $\ln N$ for $E=-2.9,-2.8, \ldots,-2$ (from up down). The solid lines are the linear fits of slope $0.91 \pm 0.01$. (c) Phase diagram of the SU (2) model for $B=1 / 2$ (red circle) and $B=1 / 5$ (black square) in the $E-W$ plane. Above $W_{c}=6.3$, all states are localized.

$i=\left(x_{i}, y_{i}\right)$ is a site on this lattice. According to our previous discussion, the wave functions for energies $|E|<$ 3.001 belong to the band of critical states. Their fractal nature can be confirmed by computing the participation ratio,

$$
P R(E) \equiv \frac{1}{N \sum_{i}\left|\psi_{E}(i)\right|^{4}},
$$

where $N$ is the number of sites. For a state whose wave function occupies a fractal space of dimension $D(E)$ [22], $P R$ scales with $N$ as $P R \propto N^{-1+D(E) / 2}$, with $D(E) \geq 0$. For a localized (metallic) state $D(E)=0[D(E)=2]$. The upper panel of Fig. 3(a) displays $P R \times N$ as a function of energy for $B=1 / 5, W=1$ (same as those in Fig. 2) and various $M$. All curves for $E<E_{c}$ (localized region) merge at $D(E)=0$, showing the independence of $P R \times N$ on $M$. Thus, $\left\{\psi_{E}(i)\right\}$ for $E<E_{c}$ are indeed localized. For $0 \geq E>E_{c}, P R \times N$ increases with $M$. To demonstrate that these states are critical with a nontrivial fractal structure, we display $\ln [P R]$ vs $\ln N$ in Fig. 3(b) for 10 different energies. The corresponding curves are virtually parallel straight lines with a common slope of $0.91 \pm 0.01$, indicating $D(E)=1.82 \pm 0.02$ for those states.

The fractal nature of the critical states is universal in the sense that $D(E)$ does not depend neither on the magnetic field (as long as it is strong enough) nor on energy (as long as $E>E_{c}$ ). This is substantiated in the bottom panel of Fig. 3(a) where $D(E)$ is plotted vs $E$ for $W=1$ and $B=1 / 5$ (red circles) and $B=1 / 2$ (blue triangles). It is instructive to compare the fractal properties of the critical wave functions discussed above with those of the wave functions for the $\mathrm{SU}(2)$ model at zero magnetic field (that is, the metallic side for the symplectic ensemble). The fractal dimension of these wave function as a function of $E$ is also shown in the bottom panel of Fig. 3(a) (black squares). In contrast, with the case $B=1 / 5$ for which the fractal dimension is shown to be $1.82 \pm 0.02$, the extended states in the absence of the field are metallic $[D(E)=2]$ and occupy the entire lattice. The fractal dimension obtained in our calculations is higher than that of the critical states in the IQHE $(D=1.75$ [23]) and in the ALT for the SU(2) model at zero field $(D=1.66 \pm$ 0.05 [24]).

The critical point $E_{c}$ that marks the edge of the band of critical states clearly depends on the strength of disorder $W$. The larger is $W$, the smaller is $E_{c}$. On the other hand, for strong enough field, $E_{c}$ is virtually independent on the magnetic field. Figure 3(c) depicts $E_{c}(W)$ vs $W$ for $B=1 / 5$ (black squares) and $B=1 / 2$ (red circles). The fact that for $W \geq 6.5$ all states are localized (albeit in the absence of SOC), has already been substantiated [20].

In conclusion, it is found that the nature of ALT for 2DEG with potential (site) disorder and SOC subject to a strong perpendicular magnetic field depends on whether the SOC is realized by constant or fully random SU(2) matrices operating in spin space. For constant SOC, there is a normal ALT on each LB, separating localized and metallic states that form a band of finite width. The corresponding critical exponent is similar to that obtained in the absence of magnetic field for the symplectic ensemble. On the other hand, for the fully random SU(2) model of the SOC, Eq. (3), the pattern of separated LBs is smeared and the system undergoes a BKT-type transition separating localized states from critical states. The localization length diverges as an exponential of an inverse square root and the critical states form a band of finite width and occupy a fractal space whose dimension is about 1.82. This is in contrast with the zero field case, where, in the presence of fully random spin-orbit scattering, the system undergoes a regular ALT separating localized states from extended (metallic) states. Interestingly, the interplay between the symplectic transition in two dimensions, and the BKT transition, has been pointed out recently [25] in the context of the surface of layered topological insulators. It may be that the model studied here is a microscopic implementation of such a theory.

This work is supported by NSFC of China Grant No. (11374249) and Hong Kong RGC Grant (No. 605413). Y.A. would like to thank the Physics Department of HKUST for hospitality during 20122014. The research of Y. A. is partially supported by the Israeli Science Foundation Grants No. 1173/2008 and No. 400/2012. 
*Corresponding author.

yshai@bgumail.bgu.ac.il

Corresponding author. phxwan@ust.hk

[1] E. P. Wigner, Group Theory and its Application to the Quantum Mechanics of Atomic Spectra (Academic Press, New York, 1959).

[2] F. J. Dyson, J. Math. Phys. (N.Y.) 3, 140 (1962); 3, 157 (1962); 3, 166 (1962).

[3] M. L. Mehta, Theory of Random Matrices, 2nd ed. (Academic Press, New York, 1991).

[4] B. Kramer and A. Mackinnon, Rep. Prog. Phys. 56, 1469 (1993).

[5] B. Huckestein, Rev. Mod. Phys. 67, 357 (1995).

[6] E. Abrahams, P. W. Anderson, D. C. Licciardello, and T. V. Ramakrishnan, Phys. Rev. Lett. 42, 673 (1979).

[7] D. Friedan, Phys. Rev. Lett. 45, 1057 (1980).

[8] S. Hikami, A. I. Larkin, and Y. Nagaoka, Prog. Theor. Phys. 63, 707 (1980).

[10] D. J. Thouless, M. Kohmoto, M. P. Nightingale, and M. den Nijs, Phys. Rev. Lett. 49, 405 (1982).

[11] H. Levine, S. B. Libby, and A. M. M. Pruisken, Nucl. Phys. B240, 30 (1984).

[12] Y. Avishai and Y. Meir, Phys. Rev. Lett. 89, 076602 (2002).
[13] V. L. Berezinskii, Sov. Phys. JETP 32, 493 (1971); J. M. Kosterlitz and D. J. Thouless, J. Phys. C 6, 1181 (1973).

[14] X. R. Wang, Phys. Rev. B 51, 9310 (1995); 53, 12035 (1996).

[15] Y. Asada, K. Slevin, and T. Ohtsuki, Phys. Rev. Lett. 89, 256601 (2002).

[16] M. M. Glazov, E. Ya. Sherman, and V. K. Dugaev, Physica (Amsterdam) 42E, 2157 (2010).

[17] O. Millo, S. J. Klepper, M. W. Keller, D. E. Prober, S. Xiong, A. D. Stone, and R. N. Sacks, Phys. Rev. Lett. 65, 1494 (1990).

[18] T. Fukui, Nucl. Phys. B575, 673 (2000).

[19] X. C. Xie, X. R. Wang, and D. Z. Liu, Phys. Rev. Lett. 80, 3563 (1998).

[20] C. Wang, Y. Avishai, Y. Meir, and X. R. Wang, Phys. Rev. B 89, 045314 (2014).

[21] W. H. Press, S. A. Teukolsky, W. T. Vetterling, and B. P. Flannery, Numerical Recipes in Fortran 77 2nd ed. (Cambridge University Press, Cambridge, England, 1996).

[22] X. R. Wang, Y. Shapir, and M. Rubinstein, Phys. Rev. A 39, 5974 (1989).

[23] F. Evers, A. Mildenberger, and A. D. Mirlin, Phys. Rev. B 67, 041303(R) (2003).

[24] H. Obuse and K. Yakubo, Phys. Rev. B 69, 125301 (2004).

[25] L. Fu and C. L. Kane, Phys. Rev. Lett. 109, 246605 (2012). 